\title{
Non-immersive Virtual Reality Exercising in Orthopaedic Rehabilitation: Qualitative Analysis Regarding Patient Experience
}

\author{
Lindsay J Millar ${ }^{1 *}$, Brian W Scarisbrick ${ }^{2}$ and Philip J Rowe ${ }^{1}$ \\ ${ }^{1}$ Department of Biomedical Engineering, University of Strathclyde, UK \\ ${ }^{2}$ Musculoskeletal Centre, Biggart Hospital, UK
}

Submission: March 08, 2017; Published: April 25, 2017

"Corresponding author: Lindsay J Millar, Department of Biomedical Engineering, University of Strathclyde, 106 Rottenrow, Glasgow, G8 0NW, UK, Tel: 07557402054; Email: 1.clarke@strath.ac.uk

\begin{abstract}
Non-immersive virtual reality feedback during rehabilitation has been shown to be applicable and acceptable for patients who have suffered a stroke. However, assessment of this type of technology has been focused on the exploratory phase and there has been limited investigation into the use of non-immersive VR feedback in routine clinical environments. Further, the majority of research has been focused on stroke rehabilitation and there has been limited assessment of feasibility and acceptability of this feedback with other patient populations. Therefore, the aim of this study was to design a non-immersive virtual reality feedback tool for routine clinical use and determine the acceptability with patients undergoing orthopaedic rehabilitation. An optical tracking based motion capture system and a visualisation development package were used to create bespoke visual feedback for three standard orthopaedic rehabilitation exercises (sit to stand, step up and weight transfer). Fifteen post-operative total knee arthroplasty patients completed rehabilitation with feedback for three of nine exercises, once a week for six weeks in an exercise class based environment. Fourteen of 15 patients completed a questionnaire which assessed comfort, enjoyment and understanding regarding use of the virtual reality tool. The majority of patients found most aspects of the tool acceptable and it was therefore concluded that non-immersive virtual reality is acceptable to patients receiving orthopaedic rehabilitation in a clinical environment.
\end{abstract}

Keywords: Visual feedback; Motion analysis; Orthopaedic rehabilitation

\section{Introduction}

The feedback of information to patients regarding their movement during rehabilitation has been described as a key aspect in achieving a desirable outcome. It has been suggested that "feedback on the performance of the action is essential for functional recovery" [1]. Knowledge of performance (KP) relies on giving patients real time feedback about their movement and may help to highlight any compensatory movements [1]. Further, it has been suggested that KP may lead to greater improvements in movement performance and quality in comparison to other types of feedback [2]. It has been suggested that providing KP in the form of augmented visual feedback using virtual reality (VR) may have a positive effect on rehabilitation outcomes [1] and there is also evidence indicating the importance of providing patients with information regarding their performance in order to learn a new task or complete an exercise [3-5]. With the introduction of more mainstream VR systems designed for video gaming, a number of authors have investigated the use of such systems to provide KP feedback during rehabilitation.
Currently, the most popular platforms are the Microsoft Kinect $^{\mathrm{TM}}$ (Microsoft UK, Berkshire) and Nintendo Wii ${ }^{\mathrm{TM}}$ (Nintendo of America, Redmond, WA, USA).The Kinect has been used to provide VR feedback for rehabilitation [6,7], although a number of studies have reported inconsistencies with kinematic measurements and participant detection [8-10] and therefore routine use of Kinect to deliver KP feedback is currently limited. A number of studies have also investigated the effectiveness of using the Wii and Wii Fit during rehabilitation [11-14]; however measurement of gross body movements is not possible which limits the effectiveness of KP feedback. Therefore, if reliable, movement based feedback is required, more sophisticated measurement systems must be employed. However, the majority of research into VR for rehabilitation with more advanced systems currently remains in the exploratory phase [15], and therefore there has been limited assessment of the feasibility and acceptability of non-immersive, movement based VR feedback in routine clinical environments. 
Traditional, camera based motion capture may be a suitable alternative to deliver KP feedback as it is currently the gold standard for measuring human movement [16,17] and is becoming more accessible in clinical environments [18]. There are currently no published cohort studies in which motion capture is used to deliver KP feedback to patients in the clinical environment. Further, any studies which used other platforms to deliver visual feedback to patients failed to report on patient experience. One study [19] did assess the acceptability of four different types of feedback on healthy participants during gait training. However, results only demonstrated which type of feedback users found most intuitive and did not report any results regarding the experience of users. Therefore, the aim of this study was to develop a tool which uses motion capture to provide accurate, real time KP feedback augmented with simple biomechanical data, in the clinical environment and determine the acceptability of such a tool with patients who are receiving orthopaedic rehabilitation.

\section{Methods}

\section{Development of visual feedback scenarios}

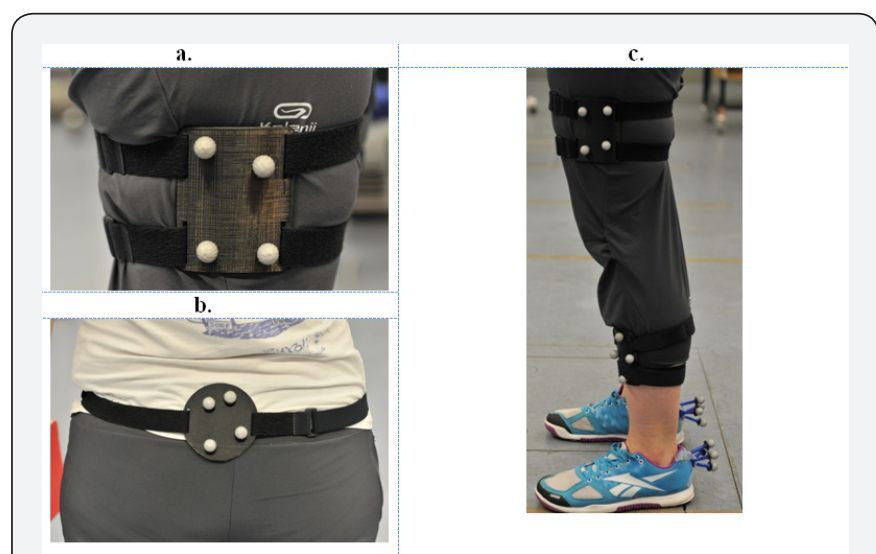

Figure 1: Strathclyde cluster model. One rigid cluster of four markers is placed on each lower limb segment. a. Thigh and shank clusters are attached using Velcro straps and placed laterally on the segment; b. Pelvis cluster is also attached using Velcro straps and placed on the back, below the level of the PSISs; c. Approximate location of thigh, shank and foot plates.

A bespoke, cluster based motion analysis protocol (Figure 1) with a pointer based calibration method (Strathclyde Cluster Model) which has been previously validated for calculation of lower limb kinematics [20] was used to develop an avatar which could demonstrate lower limb movement. Markers were tracked using an 8 camera Vicon Bonita system and accompanying Vicon Tracker software. Marker trajectories were then streamed into a bespoke application development package (D Flow; Motekforce Link, Netherlands) where kinematics were calculated and non-immersive VR feedback was provided. Previous evidence has suggested that patients are comfortable viewing only the segments of interest when using visual feedback [21] and therefore a simplified avatar comprised of the lower limbs, a trunk and a head was developed (Figure 2).

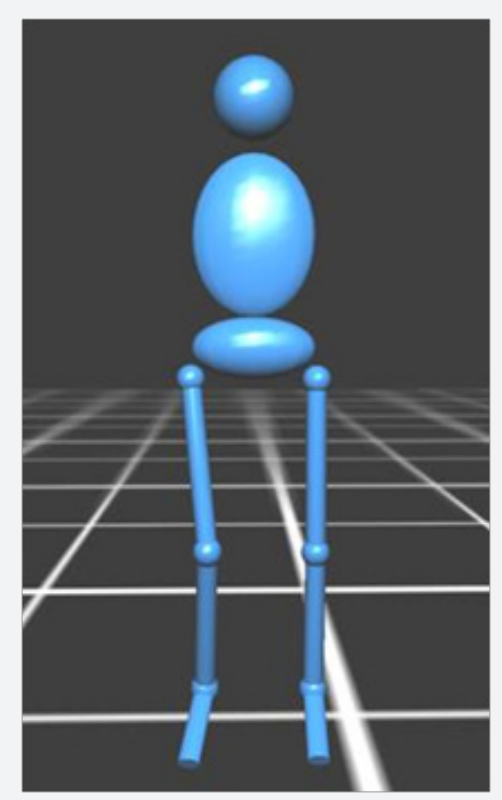

Figure 2: Avatar displayed in the virtual environment and driven by the Strathclyde Cluster Model. The Strathclyde cluster model is currently limited to the lower limbs and therefore the position of the avatar's trunk and head are driven by the position of the pelvis and are for aesthetic purposes only.

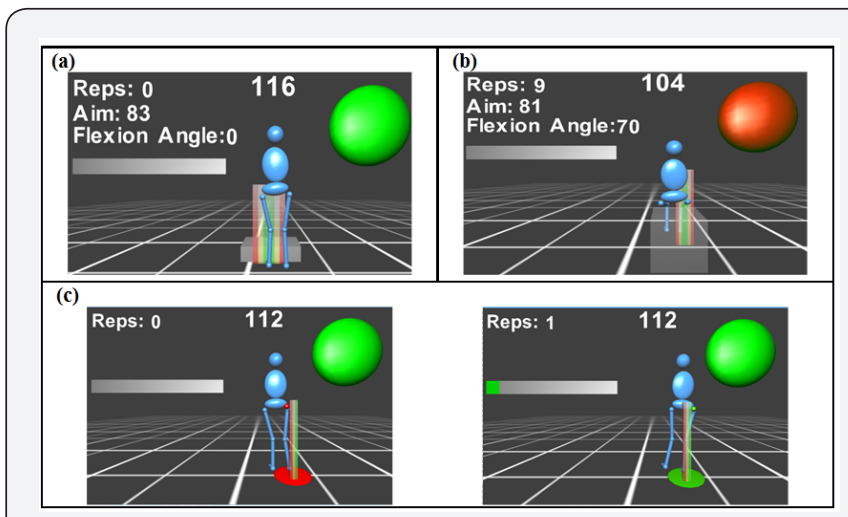

Figure 3: Examples of feedback for lower limb rehabilitation exercises (a) Step up (b) Sit to stand (c) Weight transfer. Green amber and red bands in (a) and (b) provide a threshold for medial/lateral movement of the limb and in (c) they provide an aim for the patient to move towards. Repetitions are defined as good, medium or poor which is represented by the large sphere changing to green, amber or red, respectively. Patients are provided with a goal which is defined by the range of motion of the unaffected limb. In (a) and (b) the maximum range of motion of the affected limb is measured for each repetition and presented to the patient, with the goal of getting as close to the goal as possible.

Three bespoke feedback scenarios were developed for "step up", "sit to stand" and "weight transfer" exercises (Figure 3). For the step up and sit to stand exercises, patients were provided with a "goal" which was equivalent to the range of motion (ROM) they could achieve on their unaffected side. Depending on how 
close the ROM of the affected side was to the "goal", repetitions were defined as good, medium or poor and were represented by a large sphere in the virtual environment turning green, amber or red, respectively (Figure 3). Green, amber and red bands were also used to guide the patient in limiting excessive medial or lateral movement of the affected limb. For the weight transfer exercise, the bands were used as a goal, where the aim was for the patient to move the hip of the affected side as far into the bands as possible, aiming to get to the green band each time. All feedback scenarios also had a progress indicator which filled up as a green bar every time a good repetition was performed and after 10 good repetitions the patient would receive a firework animation and motivational message. Further, upon completion of the exercises, patients were provided with the percentage of good, medium and poor reps they achieved. Feedback was controlled by a user friendly graphical user interface which allowed control over which exercise was to be performed, what was displayed in the virtual environment and which plane the patient was viewing their movement from.

\section{Qualitative feedback from patients}

This study was completed in partnership with NHS Ayrshire and Arranat the Musculoskeletal centre, Biggart Hospital, Prestwick, Scotland. Ethical approval was granted by the NHS Ethics Committee, West of Scotland REC1, Western Infirmary, Glasgow, UK. The study population consisted of patients who had undergone total knee arthroplasty and were within their first few weeks of post-operative recovery. Fifteen patients

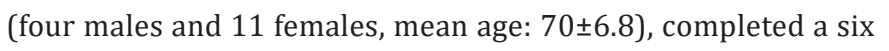
week, class based, outpatient rehabilitation program focused on increasing quadriceps strength and improving ROM at the hip and knee. Feedback was provided on a TV monitor in front of the patient for three of nine exercises using the feedback scenarios described in the previous section (Figure 4). The other six exercises were completed as normal with no feedback.

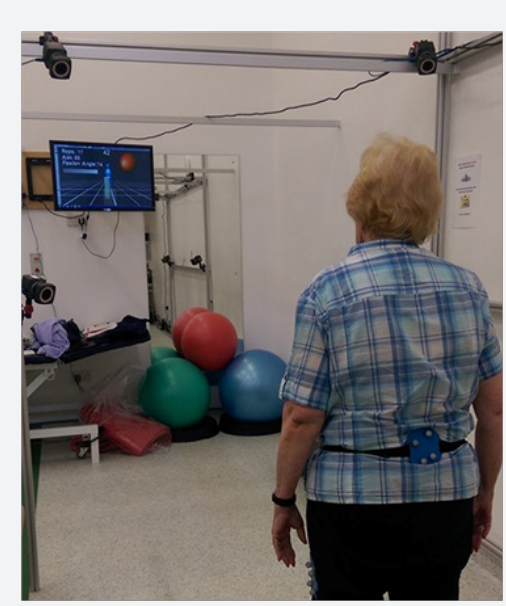

Figure 4: Patient performing rehabilitation exercises with nonimmersive VR feedback.

The feedback system took less than 5 minutes to setup and calibrate with each subject. In order to determine the acceptability of the feedback, a questionnaire was developed which aimed to determine how patients felt about wearing cluster markers and being calibrated, how they felt about seeing a virtual representation of themselves, if they understood the information which was displayed on the screen and also whether they enjoyed using the tool or not. Questionnaires were completed following the last of the six rehabilitation sessions and patients were asked to rate how much they agreed or disagreed with each statement using a five point Link ert scale. Table 1 details each statement on the questionnaire.

\section{Results}

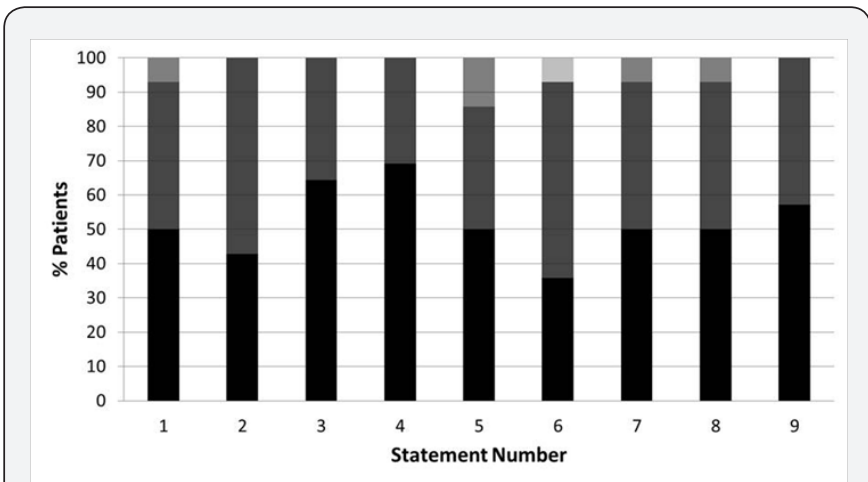

Figure 5: Results from patient feedback questionnaires. Black: strongly agree, dark grey: agree, medium grey: neither agree nor disagree, light grey: disagree.

Table 1: Statement numbers and accompanying statements in patient questionnaire.

\begin{tabular}{|c|c|}
\hline Statement Number & $\begin{array}{c}\text { Statement } \\
\text { I found wear }\end{array}$ \\
\hline 2 & $\begin{array}{c}\text { I found the calibration process } \\
\text { (palpation of bony landmarks) } \\
\text { comfortable }\end{array}$ \\
\hline 3 & $\begin{array}{c}\text { I felt comfortable seeing a virtual } \\
\text { representation of myself on the screen }\end{array}$ \\
\hline 4 & $\begin{array}{c}\text { I was comfortable aiming for a virtual } \\
\text { target }\end{array}$ \\
\hline 5 & $\begin{array}{c}\text { I understood the biomechanical } \\
\text { information which was displayed on the } \\
\text { screen }\end{array}$ \\
\hline 6 & $\begin{array}{c}\text { I found the biomechanical information } \\
\text { useful in helping me to complete the } \\
\text { exercise }\end{array}$ \\
\hline 7 & $\begin{array}{c}\text { I found the visualisations helped me } \\
\text { understand how I was moving }\end{array}$ \\
\hline 8 & $\begin{array}{c}\text { I felt that I could discuss the information } \\
\text { on the screen with my physiotherapist }\end{array}$ \\
\hline 9 & $\begin{array}{c}\text { I enjoyed using the visualisation tool as } \\
\text { part of my rehabilitation }\end{array}$ \\
\hline
\end{tabular}

Fourteen of 15 patients returned completed questionnaires following use of the feedback tool, the results of which are presented in Figure 5. The majority of patients either strongly agreed or agreed with all statements. Statement four "I was comfortable aiming for a virtual target" showed the highest 
percentage of strongly agree responses whereas statement six "I found the biomechanical information useful in helping me to complete the exercise" saw the lowest. Further, statement 6 was the only statement to receive a disagree response. In general, the patients who experienced the feedback strongly approved of it. Patients were also asked to state anything they liked or did not like about the tool. Table 2 details the responses from patients regarding use of the tool. Some responses could be interpreted as positive or negative; however, the majority of responses were positive, with a number of patients stating they found it helpful and motivating.

Table 2: Responses from patients regarding use of the VR feedback tool.

\begin{tabular}{|c|c|}
\hline Positive Responses & Negative Responses \\
\hline $\begin{array}{c}\text { "I would have liked a saved } \\
\text { record of how I was doing" }\end{array}$ & $\begin{array}{c}\text { "I would have liked a saved } \\
\text { record of how I was doing" }\end{array}$ \\
\hline $\begin{array}{c}\text { "I didn't want the machine to } \\
\text { beat me" }\end{array}$ & $\begin{array}{c}\text { "I didn't want the machine to } \\
\text { beat me" }\end{array}$ \\
\hline "I could see how I was managing" & $\begin{array}{c}\text { "I didn't really pay attention to } \\
\text { the information" }\end{array}$ \\
\hline "I can see my progress" & "It made me tired" \\
\hline "Helpful - motivating" & \\
\hline $\begin{array}{c}\text { "I liked the fact you could watch } \\
\text { your progress every week" }\end{array}$ & \\
\hline "I liked seeing the improvement" & \\
\hline $\begin{array}{c}\text { "Found it helpful in helping } \\
\text { me work towards correcting } \\
\text { movements" }\end{array}$ & \\
\hline $\begin{array}{c}\text { "Helped to motivate and } \\
\text { challenge me to do better" }\end{array}$ & \\
\hline $\begin{array}{c}\text { "A very helpful, self motivating } \\
\text { tool" }\end{array}$ & \\
\hline $\begin{array}{c}\text { "The information was like a } \\
\text { traffic light" }\end{array}$ \\
\hline $\begin{array}{c}\text { "I didn't get any green so I'll need } \\
\text { to practice that one at home" }\end{array}$ \\
\hline
\end{tabular}

\section{Discussion}

For the vast majority of statements, patients either agreed or strongly agreed although there was a small percentage of responses which were 'neither agree nor disagree' and 'disagree'. More than $50 \%$ of patients strongly agreed with statements three, "I felt comfortable seeing a virtual representation of myself on the screen" four, "I was comfortable aiming for a virtual target" and nine, "I enjoyed using the visualisation tool as part of my rehabilitation" suggesting these were the most acceptable aspects of the tool. This would suggest that most patients don't have a problem with the avatar and can relate what is happening on the screen to their own movement. Further, it suggests the majority of patients enjoyed seeing their movement in this way. There is currently little evidence to support whether patients enjoy and can accept seeing their movement as an avatar. One previous study gathered feedback from a patient focus group regarding visualisation with an avatar and the response was also positive [21]. However the majority of studies which investigate the use of visualisation and virtual reality are not yet at the stage to be tested with patients [7], therefore there is very little data on patient response to feedback of this type.

Less than $50 \%$ of patients strongly agreed with statements two "I found the calibration process (palpation of bony landmarks) comfortable" and six, "I found the biomechanical information useful in helping me to complete the exercise" suggesting these may be less strongly acceptable aspects of the tool. Statement two was regarding the calibration process and whether patients found it comfortable, so perhaps, in future, steps should be taken to improve the calibration process to make it more comfortable for patients. The lower percentage of 'strongly agree' responses for this statement could have been due to the periods of standing required for the calibration process, particularly at the baseline stage where patients may only be a few days post op, or due to the palpation of bony landmarks which may have been uncomfortable, particularly on the affected knee. The introduction of functional calibration would reduce the uncomfortable palpation of landmarks, however it may not eliminate periods of standing and may also require patients to perform ROM tasks which they may find even more uncomfortable than simply standing for a few minutes [22]. The largest proportion of 'neither agree nor disagree' was for statement five "I understood the biomechanical information which was displayed on the screen". This suggests that either the term biomechanical was poorly understood by the users or the information aspect of the tool may need improvement. Uptake of the biomechanical feedback was entirely dependent on the individual patient; therefore further questioning would be required to gauge the numbers of patients who paid close attention to the information, and those who didn't.

A number of patients also felt less strongly about being able to discuss the information with their physiotherapist. There could be a number of reasons for this. First, some patients said they felt less strongly about being able to understand the information which may have been a reason they wouldn't want to discuss it with their physiotherapist. Second, there was only one physiotherapist for a class full of patients so it may have been that there wasn't time for every patient to discuss every exercise with the physiotherapist. In future, the tool could include a virtual teacher giving visual and verbal feedback to the user during and at the end of each activity. Alternatively the tool could be used in a one to one environment where the physiotherapist can dedicate all their attention to the patient to ensure they understand the information and can answer any questions the patient may have. Virtual teachers are becoming common in other IT scenarios but have not as yet been used in rehabilitation support. A number of studies using VR feedback have observed positive results when providing subjects with a virtual example of how the task should be carried out $[5,23]$. Unlike the current study, Holden \& Todorov [23,5], only presented participants with a virtual teacher and no biomechanical information. This 
suggests that it may be more useful to provide a virtual teacher which demonstrates the appropriate ROM for each patient alongside the patient's own movement. This may increase the likelihood that patients understand the information and may also increase their willingness to pay attention to the screen, as they have something which they need to copy.

There are a limited number of studies which have used visual feedback during rehabilitation, and of these studies, none appear to have reported qualitative feedback from patients regarding their experience. One study [19] did assess the acceptability of 4 different types of feedback on healthy participants during gait training. The feedback ranged from a colour coded traffic light system, to a real time graph of the parameter of interest. There was no significant difference between ratings for each type of feedback although the colour coded feedback was rated "most intuitive" when patients were asked to modify their knee adduction moment. The current study did not investigate the effects of different types of feedback, although the positive response to a colour coded system seems to be present in both studies. Further, limited studies have assessed the feasibility and acceptability of VR systems based on sophisticated measurement devices in a routine clinical environment. This study successfully implemented routine VR feedback within a clinical rehabilitation class with minimal disruption to the standard process of care, therefore proving that routine VR feedback with sophisticated measurement systems is possible in the clinical environment.

\section{Conclusion}

Patients were comfortable seeing a virtual representation of themselves which agrees with previous studies which used VR feedback in the form of an avatar. Further, patients were comfortable wearing the cluster markers and undergoing the calibration process and they enjoyed using the feedback tool as part of their rehabilitation. However, some improvements could be made regarding the biomechanical information which was displayed. In future, it may be helpful to convey the information in a clearer manner, perhaps using a virtual teacher, so that all patients can understand and follow it. Further, routine use of a VR feedback system driven by motion capture was shown to be feasible in the clinical environment which indicates the possibility of extending the use of sophisticated measurement systems for VR applications beyond research environments.

\section{Conflict of Interest Statement}

The authors declare there is no conflict of interest.

\section{References}

1. Jones L, van Wijck F, Grealy M, Rowe P (2011) A changing stroke rehabilitation environment: implications for upper limb interventions. In: 5th International Conference on Pervasive Computing Technologies for Healthcare and Workshops pp.04-23.

2. Subramanian SK, Massie CL, Malcolm MP, Levin MF, (2010) Does Provision of Extrinsic Feedback Result in Improved Motor Learning in the Upper Limb Poststroke? A Systematic Review of the Evidence.
Neurorehabil Neural Repair 24(2): 113-124.

3. Levinger P, Zeina D, Teshome AK, Skinner E, Begg R, et al. (2016) A real time biofeedback using Kinect and Wii to improve gait for post-total knee replacement rehabilitation: a case study report. Disabil Rehabil Assist Technol 11(3): 251-262.

4. Swinnen S (1996) Information Feedback for Motor Skill Learning: A review, in: Advances in Motor Learning and Control. Human Kinetics.

5. Todorov E, Shadmehr R, Bizzi E (1997) Augmented Feedback Presented in a Virtual Environment Accelerates Learning of a Difficult Motor Task. J Mot Behav 29(2): 147-158.

6. Lozano-Quilis JA, Gil-Gómez H, Gil-Gómez JA, Albiol-Pérez S, PalaciosNavarro G, et al. (2014) Virtual Rehabilitation for Multiple Sclerosis Using a Kinect-Based System: Randomized Controlled Trial. JMIR Serious Games 2(2): e12.

7. Webster D, Celik O (2014) Systematic review of Kinect applications in elderly care and stroke rehabilitation. J Neuroeng Rehabil 11: 108.

8. Baldewijns G, Verheyden G, Vanrumste B, Croonenborghs T (2014) Validation of the kinect for gait analysis using the GAITRite walkway, Conf Proc IEEE Eng Med Biol Soc pp. 5920-5923.

9. Clark RA, Pua YH, Fortin K, Ritchie C, Webster KE, et al. (2012) Validity of the Microsoft Kinect for assessment of postural control. Gait Posture 36(3): 372-377.

10. Xu X, McGorry RW (2015) the validity of the first and second generation Microsoft KinectTM for identifying joint center locations during static postures. Appl Ergon 49: 47-54.

11. Baltaci G, Harput G, Haksever B, Ulusoy B, Ozer H, et al. (2012) Comparison between Nintendo Wii Fit and conventional rehabilitation on functional performance outcomes after hamstring anterior cruciate ligament reconstruction: prospective, randomized, controlled, doubleblind clinical trial. Knee Surg Sports Traumatol Arthrosc 21(4): 880887.

12. Chao YY, Scherer YK, Montgomery CA (2015) Effects of Using Nintendo WiiTM Exergames in Older Adults A Review of the Literature. J Aging Health 27(3): 379-402.

13. Fung V, Ho A, Shaffer J, Chung E, Gomez M, et al. (2012) Use of Nintendo Wii FitTM in the rehabilitation of outpatients following total knee replacement: a preliminary randomised controlled trial. Physiotherapy 98(3): 183-188.

14. Park J, Lee D, Lee S (2014) Effect of Virtual Reality Exercise Using the Nintendo Wii Fit on Muscle Activities of the Trunk and Lower Extremities of Normal Adults. J Phys Ther Sci 26(2): 271-273.

15. Proffitt R, Lange B (2015) Considerations in the Efficacy and Effectiveness of Virtual Reality Interventions for Stroke Rehabilitation: Moving the Field Forward. Phys Ther 95(3): 441-448.

16. Cook RE, Schneider I, Hazlewood ME, Hillman SJ, Robb JE, et al. (2003) Gait analysis alters decision-making in cerebral palsy. J Pediatr Orthop 23(3): 292-295.

17. Gage JR (1993) Gait analysis. An essential tool in the treatment of cerebral palsy. Clin Orthop Relat Res 288: 126-134.

18. Carse B, Meadows B, Bowers R, Rowe P (2013) Affordable clinical gait analysis: An assessment of the marker tracking accuracy of a new lowcost optical 3D motion analysis system. Physiotherapy 99(4): 347-351.

19. Noort van den JC, Steenbrink F, Roeles S, Harlaar J (2015) Realtime visual feedback for gait retraining: toward application in knee osteoarthritis. Med Biol Eng Comput 53(3): 275-286.

20. Millar LJ, Murphy AJ, Rowe PJ (2015) Comparison of a bespoke biomechanical model to the industry gold standard for calculation of lower limb kinematics during walking. Gait \& Posture 42(1): S78. 
21. Loudon D, Macdonald AS, Carse B, Thikey H, Jones L, et al. (2012) Developing visualisation software for rehabilitation: Investigating the requirements of patients, therapists and the rehabilitation process Health Informatics J 18(3): 171-180.

22. Piazza SJ, Okita N, Cavanagh PR (2001) Accuracy of the functional method of hip joint center location: effects of limited motion and varied implementation. J Biomech 34(7): 967-973.

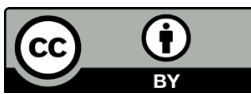

This work is licensed under Creative Commons Attribution 4.0 License

DOI: $10.19080 /$ CTBEB.2016.01.555610
23. Holden M, Todorov E (1999) Virtual Environment Training Improves Motor Performance in Two Patients with Stroke: Case Report. Journal of Neurologic Physical Therapy 23(2): 57-67.

\section{Your next submission with Juniper Publishers} will reach you the below assets

- Quality Editorial service

- Swift Peer Review

- Reprints availability

- E-prints Service

- Manuscript Podcast for convenient understanding

- Global attainment for your research

- Manuscript accessibility in different formats

( Pdf, E-pub, Full Text, Audio)

- Unceasing customer service

Track the below URL for one-step submission https://juniperpublishers.com/online-submission.php 